tinins (Tab. 3) bzw. aller gelösten Urinbestandteile oder eine entsprechende aus der Regressionsgleichung abgeleitete Größe - der Unsicherheitsfaktor zu groß ist. Man darf darüber hinaus nicht übersehen, daß die $\mathrm{Ab}$ hängigkeit der Ausscheidung von der Tageszeit, über die unsere Beobachtungen nichts aussagen, bei diesem Problem eine große Rolle spielt.

Daher hat es auch wenig Sinn, die Steroidausscheidung pro Gramm Kreatinin auszudrücken. Wie Tabelle 3 zeigt, sind die Schwankungsbreiten dieses Parameters durchaus nicht kleiner als die der 24-Stdn.-Mengen. Aus dem Gesagten geht hervor, daß die möglichst genaue Bestimmung der Sammelperiode sowie verlustloses Sammeln des Urins weiterhin als unerläßliche Voraussetzungen zum Erzielen sinnvoller Analysenresultate anzusehen sind.
Die geringfügige, wenn auch statistisch gesicherte $\mathrm{Zu}$ nahme der Steroidausscheidung mit dem Urinvolumen konnte nur auf Grund der extremen Versucbsbedingungen erkannt werden. Diese Zunahme dürfte jedoch im normalen Schwankungsbereich der Flüssigkeitsmenge zu vernachlässigen sein. Aus diesen Gründen kommt dem Phänomen, trotz seiner statistischen Sicherung, keine praktische Bedeutung zu. Hier zeigt sich erneut, daß bei der Interpretation biologischer Erscheinungen die statistische Signifikanz sehr wohl von der Relevanz des beobachteten Effektes unterschieden werden muß. Diese Feststellung schließt jedoch keineswegs die Frage aus, ob der hier beobachtete Zusammenhang auf einem Stoffwechselphänomen oder auf einer erhöhten Sekretion beruht. Bei solchen Überlegungen wird die Rolle der Niere zu berücksichtigen sein.

\title{
Literatur
}

1. Kassenaar, A., J. Huis in't Veld, P. Siderius, H. C. Seldenrath und A. Querido, Acta endocr., K'hvn. 4; 79 (1950). - 2. Hoxt, R. E. und M. G. Levine, J. Clin. Endocr., Springfield 10, 101 (1950). - 3. LuGG, J. W. H. und J. M. Bowness, Austral. J. exp. Biol. med. Sci. 35, 395 (1957). - 4. Hamburger, C., Acta endocr., K'hvn. 17, 116 (1954). - 5. Devis, R., Bruxelles-méd. 29, 1568 (1949). - 6. Hollander, F., B. KRISS, E. KLEMpNer und R. T. Frank, Endocrinology 33, 217 (1943). - 7. McHeNRY, E. W., E. M. Semmons, R. Pearse und E. G. Meyer, Cancer Res. 7, 534 (1947). - 8. Laroche, G., A. Corteel und J. Delor, Ann. endocr., Paris 7, 191 (1946). - 9. Norymberski, J. K., R. D. Stubbs und H. F. West, Lancet, London I, 1276 (1953). 10. Callow, N. H., R. K. Callow und C. W. Emmens, Biochem. J. 32, 1312 (1938). - 11. Talbot, N. B., R. A. Berman und E. A. Mclachlan, J. biol. Chemistry 143, 211 (1942). - 12. Appleby, J. I., G. Gibson, J. K. Norymberski und R. D. Stubbs, Biochem. J. 60, 453 (1955). - 13. Popper, H., E. MANDel und H. Mayer, Biochem. Z. 291, 354 (1937). - 14. LindER, A., Statistische Methoden, 3. Aufl., Birkhäuser, Basel (1960). - 15. MEDGYESI, G. und K. Katona, Ärztl. Lab. 7, 247 (1961).

Professor Dr. R. Borth St. Michael's Hospital Toronto 2, Ontario Canada Professor Dr. H. Breuer 53 Bonn-Venusberg

\section{Photometrische Bestimmung der „klassischen“ Leucinaminopeptidase im Blutplasma und -serum}

\author{
Von R. J. HASChEN, W. FARR und D. Reichelt \\ Aus dem Institut für Klinische Biochemie der Martin-Lutber-Universität, Halle/Saale \\ (Direktor: Prof. Dr. med. R.J. Hascben)
}

(Eingegangen am 14. November 1966)

Es wird eine neue photometrische Methode zur Bestimmung der Leucinaminopeptidase („LAP“) im Serum beschrieben, die aufgrund ihrer Empfindlichkeit und Einfachheit für Routinezwecke geeignet ist. Als Substrat dient DL-Leucinhydrazid, das durch Serum-LAP bei pH 10,0 optimal zu Hydrazin und L-Leucin hydrolysiert wird. Das freigesetzte Hydrazin wird mit p-Dimethylaminobenzaldehyd in salzsaurer Lösung zu einem orangeroten Farbsalz umgesetzt, dessen Konzentration der enzymatischen Aktivität proportional ist und bei $455 \mathrm{~nm}$ photometriert wird. Die Bestimmung bei $\mathrm{pH} \mathrm{9,25} \mathrm{mit} \mathrm{optimaler} \mathrm{Mg}^{2+}$-Aktivierung ist wegen des größeren Zeitaufwandes für Routinezwecke weniger geeignet. - Die Normalwerte der LAP-Aktivität wurden im Serum und Plasma von Blutspendern bestimmt.

A new photometric method is described for the determination of leucine aminopeptidase ("LAP") in serum. It is sufficiently sensitive and simple for routine purposes. The substrate is DL-leucine hydrazide, which is hydrolysed by serum LAP optimally at $\mathrm{pH} 10.0$ to hydrazine and L-leucine. The released hydrazine is reacted with p-dimethylamino-benzaldehyde in hydrochloric acid solution, to give an orange-red salt. The concentration of the salt is proportional to the enzymic activity, and it is measured photometrically by its absorption at $455 \mathrm{~nm}$. At $\mathrm{pH} 9.25$ with optimal $\mathrm{Mg}^{2+}$-activation, longer time is required and the method is less suitable for routine measurements. The normal levels of LAP were determined in the serum and plasma from blood banks.

Die Leucinaminopeptidase „LAP“ (L-Leucylpeptidhydrolase, EC 3.4.1.1) des Serums scheint nach bisherigen Erfahrungen $(1,2,3)$ ein empfindlicher Indikator von Leberparenchymschäden zu sein. Von dieser „,klassischen " LAP (4) ist die Aminosäure-Arylamidase (5-9) zu unterscheiden, die in vielen Arbeiten zu Unrecht als
LAP bezeichnet wurde. Sie wurde vor allem bei intraund extrahepatischer Obstruktion erhöht gefunden (10). Als spezifisches Substrat der LAP gilt L-Leucinamid (4). Leucin- und vor allem Alanin-p-nitroanilid (11) und $-\beta$-naphthylamid (12) werden dagegen vorwiegend oder ausschließlich von der Aminosäure-Arylamidase hydro- 
lysiert (9). Leucin-p-nitroanilid ist auch deshalb zur Bestimmung der LAP ungeeignet, weil es beim $\mathrm{pH}-$ Optimum dieses Enzyms eine beträchtliche Spontanhydrolyse erleidet (13). Die "klassische“ LAP kann prinzipiell mit folgenden Methoden bestimmt werden: Titration in Alkohol (14); Titration in Aceton (15); Quantitative Papierchromatographie (16-18); Mikrodiffusion (19) und Bestimmung des freigesetzten Ammoniaks mittels des optischen Tests (20). Bei Verwendung des weniger spezifischen L-Leucylglycins als Substrat kann man auch den Zuwachs an Aminostickstoff mit der Ninhydrin-Methode messen (21).

Alle diese Methoden sind mehr oder weniger umständlich. Verwendet man dagegen anstelle von Leucinamid Leucinhydrazid, so läßt sich eine einfache und empfindliche photometrische Methode entwickeln, die an Spezifität den mit Leucinamid arbeitenden Verfahren nicht nachsteht.

\section{Methodik}

Prinzip

Die Leucinaminopeptidase der menschlichen Leber vermag die Hydrolyse von DL-Leucinhydrazid zu L-Leucin und Hydrazin zu katalysieren:<smiles>CC(C)CC(N)C(=O)NN</smiles><smiles>CC(C)CC(N)C(=O)O</smiles>

Das freigesetzte Hydrazin bildet mit p-Dimethylaminobenzaldehyd unter beidseitiger Kondensation einen gelben Farbstoff folgender Zusammensetzung (22):<smiles>CN(C)c1ccc(C=O)cc1</smiles>

Hydrazin p-Dimethylaminobenzaldehyd<smiles>CN(C)c1ccc(/C=N\N=C/c2ccc(N(C)C)cc2)cc1</smiles>

4,4'-Dimethylamino-benzaldazin

Dieser Farbstoff wird durch Zugabe von Salzsäure, die gleichzeitig die enzymatische Reaktion unterbricht, in ein orangerotes Farbsalz chinoider Natur umgewandelt $(23,24)$ :<smiles>CCOC(=O)OC/N=C/c1ccc(N(C)C)cc1</smiles>

Die der LAP-Aktivität proportionale Extinktion des Farbsalzes wird am Absorptionsmaximum von $455 \mathrm{~nm}$ gemessen (Abb. 1).

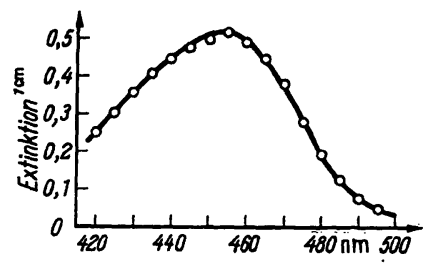

Abb. 1

Absorptionsspektrum des chinoiden Farbsalzes in $0,08 \mathrm{~N} \mathrm{HCl}$ $11,3 \cdot 10^{-0} \mathrm{M}$ Hydrazin im Meßansatz, Schichtdicke $1 \mathrm{~cm}$ (,Spekol“", VEB Carl Zeiss, Jena)

Chemikalien

Substrat

Darstellung von DL-Leucinhydrazid (26-28):

0,1 Mol DL-Leucinäthylester, frisch freigesetzt aus DL-Leucinäthylesterhydrochlorid mit Triäthylamin (29), wurde in absol. Äthanol mit 0,15 Mol Hydrazinhydrat (85proz:) 6 Stdn. am Rückfluß gekocht. Das Reaktionsprodukt wurde im Vakuum zur Trockne eingedampft, der Rückstand in absol. Äthanol aufgenommen und wiederholt eingedampft, bis das überschüssige Hydrazinhydrat beseitigt war. Der Rückstand wurde zuerst aus absol. Åthanol/absol. Äther, dann aus absol. Isopropanol/Cyclohexan umkristallisiert.

Ausbeute: $51 \%$ der Theorie. Fp.: $85-86^{\circ}$; Lit.-Fp.: $87^{\circ}$ (28). $\mathrm{C}_{5} \mathrm{H}_{15} \mathrm{~N}_{3} \mathrm{O}$ (Mol.-Gew.: 145,2); N, ber.: 28,94\%, gef.: $28,89 \%$. Das erhaltene DL-Leucinhydrazid war dünnschichtchromatographisch rein (Kieselgel G „Merck“, Laufmittel: 96proz. Athanol/ $\mathrm{H}_{2} \mathrm{O}$ (63:37); Anfärbung mit 0,5proz. Ninhydrin in $\mathrm{H}_{2} \mathrm{O}$-gesättigtem n-Butanol mit 0,7\% Eisessig).

Von diesem Substrat wurden folgende Stammlösungen hergestellt und bei $-18^{\circ}$ aufbewahrt:

Stammlösung A: $224 \mathrm{~mm}$ ( $325 \mathrm{mg} / 10 \mathrm{ml}$ aqua bidest.),

Stammlösung B: $160 \mathrm{mM}(232 \mathrm{mg} / 10 \mathrm{ml}$ aqua bidest.) für Ansätze mit $\mathrm{Mg}^{2+}-$ Zusatz.

Auf eine Herstellung des L-Leucinhydrazids wutde aus folgenden Gründen verzichtet: Eine Hemmung durch die D-Komponente ist nicht anzunehmen, da sich die Michaelis-Konstanten für DL-Leucinhydrazid (bezogen auf die L-Komponente, s. u.) und L-Leucinamid $(18,30)$ weitgehend entsprechen. Weiter entfällt bei der Synthese der DL-Verbindung die Einführung einer Amino-Schutzgruppe und der Syntheseweg wird dadurch erheblich vereinfacht, $d . h$. man benötigt billigere Ausgangs- und Endprodukte.

\section{Puffer}

Es wurde ein 0,2M Monoäthanolaminpuffer (25) (final 0,114M) vom pH 10,15 (final $\mathrm{pH} 10,0$ ) verwendet.

Herstellung: Lösung I: 0,4M Monoäthanolamin-HCl-Stammlösung $(39,04 \mathrm{~g} / l)$;

Lösung II: $0,4 \mathrm{~N} \mathrm{NaOH}$

$50 \mathrm{~m} l$ Lösung I und $44 \mathrm{~m} l$ Lösung II werden gemischt und nach $\mathrm{pH}-$ Kontrolle mit aqua bidest. auf $100 \mathrm{~m} /$ aufgefüllt.

Für die $\mathrm{pH}-\mathrm{Kurven}$ wurde ein 0,2M Monoäthanolamin/Triäthanolamin-Puffer (25) verwendet. Bei der Bestimmung der Serum-LAP unter Zusatz von $\mathrm{Mg}^{2+}$-Ionen wurde ein $0,25 \mathrm{M}$ Monoäthanolaminpuffer (25) (final 0,125M) vom pH 9,25 (final $\mathrm{pH} 9,25$ ) benutzt. Die Hydrochloride von Mono- und Triäthanolamin wurden aus den freien Basen nach deren Destillation hergestellt (25).

Substrat-Puffer-Lösung

Für eine Serie von Ansätzen wurden Puffer und Substrat-Stammlösung $A$ vorher im Verhältnis $4+1$ gemischt. Das Substrat lạ̈ßt 
sich sowohl in wäßr. Lösung als auch im Gemisch mit Puffer etwa 14 Tage im eingefrorenen Zustand aufbewahren, ohne daß eine crhebliche Eigenspaltung eintritt (s. Abb. 2).

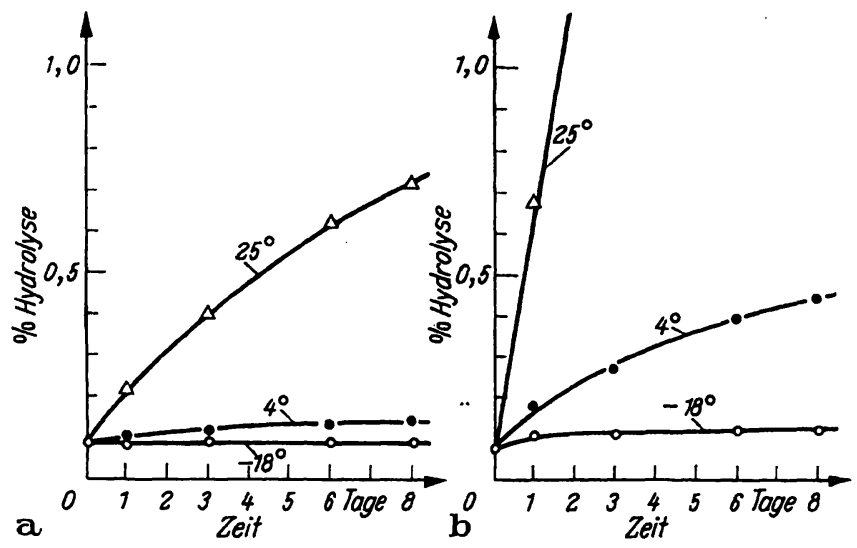

Abb. 2

Hydrolyse von $32 \mathrm{mM}$ DL-Leucinhydrazid unter verschiedenen Aufbewahrungsbedingungen

a) in wäßriger Lösung, b) in 0,2 M Monoäthanolaminpuffer $\mathrm{pH} 10,15$

\section{Puffer-Aktivator-Lösung}

I. $0,25 \mathrm{M}$ Monoäthanolamin-Puffer, $\mathrm{pH} 9,25$

II. $40 \mathrm{~m} \mathrm{M} \mathrm{MgCl} \cdot 6 \mathrm{H}_{2} \mathrm{O}$-Lösung $(0,8133 \mathrm{~g} / 100 \mathrm{ml}$ aqua bidest). Für die Bestimmung der Serum-LAP mit Zusatz von $\mathbf{M g}^{2+}$-Ionen werden I und II vorher im Verhältnis $5+1$ gemischt.

\section{Reagenz}

Die Herstellung des Reagenzes erfolgte in Anlehnung an eine Vorschrift von Kallos und Mitarbeitern $(31,32)$.

I. Lösung von $4 \mathrm{~g}$ p-Dimethylaminobenzaldehyd in $100 \mathrm{ml}$ 96 proz Åthanol oder Methanol.

II. 0,1N Salzsäure (s. Abb. 3).

Gebrauchslösung: I und II werden jeweils vor der Verwendung im Verhältnis $1+17$ gemischt.

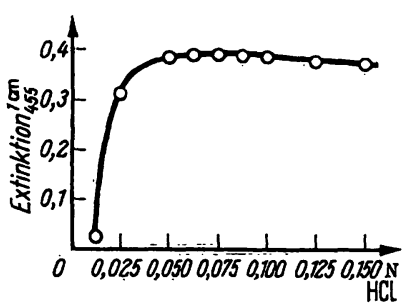

Abb. 3

Abhängigkeit der Extinktion des chinoiden Farbsalzes von der HCl-Konzentration

Die Messung erfolgte unter Ansatzbedingungen bei pH 10,0. Absžisse: Die Angaben beziehen sich auf die in den Meßansätzen vorliegenden Endkonzentrationen

\section{Arbeitsvorschrift}

Elektrophoretische 1solierung von $L A P$ und Aminosäure-Arylamidase aus Leberhomogenat

Das aus menschlicher Leber hergestellte Homogenat wurde im Stärkeblock elektrophoretisch aufgetrennt. Der Stärkeblock wurde dann in Richtung zur Anode in 19 Fraktionen von $1 \mathrm{~cm}$ Breite zerschnitten und jeweils mit $1 \mathrm{~m} l$ aqua bidest. eluiert. Die Enzymaktivität dieser Eluate wurde gegenüber DL-Alanyl- $\beta$-naphthylamid (Aminosäure-Arylamidase) und DL-Leucinhydrazid (LAP) untersucht (9) (Abb. 4).

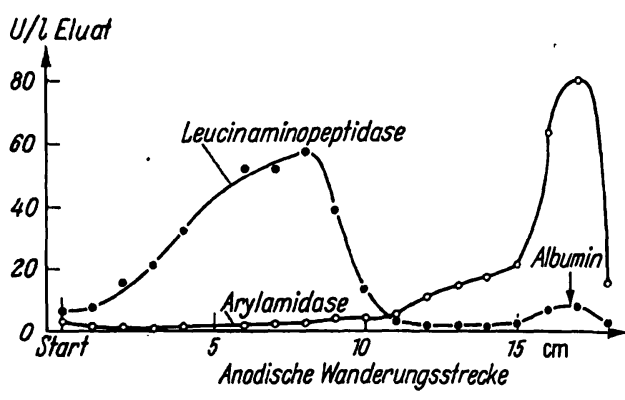

Abb. 4

Elektrophoretische Auftrennung von Arylamidase und Leucinaminopeptidase aus menschlicher Leber im Stärkeblock (Veronal$\mathrm{Na} / \mathrm{HCl}$-Puffer pH 8,6, $\mu=0,05,6-7 \mathrm{~V} / \mathrm{cm}, 16 \mathrm{Stdn}$. bei $4^{\circ}$ )

Substrate: DL-Alanyl- $\beta$-naphthylamid $(1 \mathrm{mM}$, bezogen auf L-Komponente) für Arylamidase; DL-Leucinhydrazid (1 $\mathrm{mM}$, bezogen auf L-Komponente) für LAP

\section{Gewinnung von Serum und Plasma}

Die Blutproben wurden $15 \mathrm{Min}$. nach der Blutentnahme $15 \mathrm{Min}$. bei $4500 \mathrm{~g}$ in vorgekühlten Einsätzen zentrifugiert und das Serum vorsichtig abgehoben.

Für die Plasmagewinnung wurden die Röhrchen $(10 \mathrm{ml})$ vor der Blutentnahme mit zwei Tropfen Heparin "Richter" (Budapest) versetzt.

Als Modell für ein pathologisches Serum mit hoher LAP-Aktivität wurde Normalserum verwendet, das durch Zusatz von elektrophoretisch isolierter LAP auf eine hohe Enzymaktivität gebracht worden war.

Bestimmung obne $\mathrm{Mg}^{2+}-$ Zusatz

\begin{tabular}{|c|c|c|}
\hline & Hauptwert & Leerwert \\
\hline Substrat-Puffer-Lösung & \multicolumn{2}{|c|}{$\begin{array}{l}0,5 \mathrm{~m} l \\
\text { auf } 25^{\circ} \text { temperieren }\end{array}$} \\
\hline Serum & \multicolumn{2}{|c|}{$\begin{array}{l}0,2 \mathrm{ml} \\
60 \mathrm{Min} \text {. bei } 25^{\circ} \text { inkubieren }\end{array}$} \\
\hline Farbreagenz & $\begin{array}{l}\qquad 5,0 \mathrm{ml} \\
\text { mittels Fortun } \\
\text { tige Durchmis }\end{array}$ & $\begin{array}{l}5,0 \mathrm{ml} \\
\text { atte (gleichzei- } \\
\text { g) hinzugeben }\end{array}$ \\
\hline Serum & - & $0,2 \mathrm{ml}$ \\
\hline
\end{tabular}

Mindestens 30 Min. zur Farbentwicklung bei $25^{\circ}$ belassen (s. Tab. 1); dann beide Ansätze bei $455 \mathrm{~nm}$ und d = $1 \mathrm{~cm}$ gegen das Reagenz messen.

Bestimmung mit $\mathrm{Mg}^{2+}$-Aktivierung

\begin{tabular}{lcc}
\hline & Hauptwert & Leerwert \\
\hline Puffer-Aktivator-Lösung & $0,3 \mathrm{~m} l$ & $0,3 \mathrm{~m} l$ \\
Serum & $0,1 \mathrm{ml}$ & \\
& $2 \mathrm{Stdn}$. bei $25^{\circ}$ & präinkubieren \\
Substrat-Stammlösung B & $0,1 \mathrm{~m} l$ & $0,1 \mathrm{~m} l$ \\
& $30 \mathrm{Min}$. bei $25^{\circ}$ inkubieren \\
Farbreagenz & $4,5 \mathrm{ml}$ & $4,5 \mathrm{~m} l$ \\
& wie oben zugeben \\
Serum & - & $0,1 \mathrm{~m} l$ \\
\hline
\end{tabular}

Farbentwicklung und Messung wie oben. 
Tab. 1

Ermittlung der Zeitdauer bis zur vollständigen Entwicklung des Farbstoffes bei zwei Hydrazinsulfatlösungen unterschiedlicher Konzentration

\begin{tabular}{|c|c|c|}
\hline \multirow[b]{2}{*}{$\begin{array}{c}\text { Min. nach } \\
\text { Reagenzzugabe }\end{array}$} & \multicolumn{2}{|c|}{$\mathrm{E}^{1 \mathrm{~cm}}$} \\
\hline & $\begin{array}{c}8,5 \cdot 10^{-8} M \\
\text { Hydrazin }\end{array}$ & $\begin{array}{c}3,1 \cdot 10^{-8} M \\
\text { Hydrazin }\end{array}$ \\
\hline $\begin{array}{r}5 \\
10 \\
15 \\
20 \\
25 \\
30 \\
35\end{array}$ & $\begin{array}{l}0,205 \\
0,295 \\
0,340 \\
0,375 \\
0,385 \\
0,385 \\
0,385\end{array}$ & $\begin{array}{l}1,120 \\
1,28 \\
1,36 \\
1,40 \\
1,43 \\
1,43 \\
1,43\end{array}$ \\
\hline
\end{tabular}

Anmerkungen

Nach Zugabe des Farbreagenzes nimmt die Farbintensität von Haupt- und Leerwert auch nach Ablauf von 30 Min. infolge nichtenzymatischer Hydrolyse langsam zu. Det $\Delta \mathrm{E}$-Wert bleibt aber praktisch unverändert (s. Tab. 2).

Tab. 2

Extinktionszunahmen von Haupt- und Leerwert als Funktion der Zeit

\begin{tabular}{cccc}
\hline \multirow{2}{*}{$\begin{array}{c}\text { Min. nach } \\
\text { Reagenzzugabe }\end{array}$} & Hauptwert & Leerwert & \multicolumn{1}{c}{$\Delta \mathrm{E}_{\mathbf{4 5 5}}^{1 \mathrm{~cm}}$} \\
\hline 30 & 0,238 & 0,142 & \\
90 & 0,278 & 0,180 & 0,096 \\
180 & 0,300 & 0,203 & 0,098 \\
300 & 0,330 & 0,232 & 0,097 \\
\hline
\end{tabular}

Bei Aktivitäten über $10 \mathrm{U} / l$ wird die Inkubationsdauer auf $10 \mathrm{Min}$. verkürzt und evtl. bei $0,5 \mathrm{~cm}$ Schichtdicke gemessen.

Eine Verdünnung des Serums mit aqua bidest. oder 0,9 proz. $\mathrm{NaCl}-\mathrm{Lösung}$ ist unbedingt $\mathrm{zu}$ vermeiden (s. Abb. 10e).

\section{Eicbung}

Aus einer $0,2 \mathrm{~mm}$ Hydrazinsulfatlösung ( $26 \mathrm{mg} / l$ aqua bidest.) wurde eine Verdünnungsreihe hergestellt (Hydrazinsulfat von $\mathbf{F a}$. „VEB Berlin-Chemie“). Abbildung 5 zeigt die Gültigkeit des

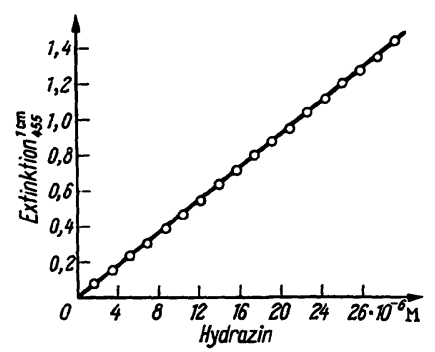

Abb. 5

Abhängigkeit der Farbintensität von der Hydrazinkonzentration (,Spekol“, VEB Carl Zeiss, Jena)

LAMBERT-BEERschen Gesetzes für den gemessenen Konzentrationsbereich. Der millimolare Extinktionskoeffizient $(\varepsilon)$ des Farbstoffes in $0,08 \mathrm{~N} \mathrm{HCl}$ beträgt:

$45,63 \mathrm{~mm}^{-1} \mathrm{~cm}^{-1}$ bei $455 \mathrm{~nm}$ (spektrale Halbwertsbreite $11 \mathrm{~nm}$ ), $42,79 \mathrm{~mm}^{-1} \mathrm{~cm}^{-1}$ bei $436 \mathrm{~nm}$ (Hg-Linie), $40,7 \mathrm{~mm}^{-1} \mathrm{~cm}^{-1}$ bei $455 \mathrm{~nm}, 0,15 \mathrm{~mm}$ Spaltbreite.

\section{Berecbnung der Enzymkonzentration}

Die LAP-Aktivität wird in Einheiten - Units (33) - angegeben. Die Enzymkonzentration errechnet sich unter Zugrundelegung einer Kinetik nullter Ordnung nach folgender Formel:

$$
\text { Enzymkonzentration }=\frac{\Delta \mathrm{E} \cdot \mathrm{V}_{\mathrm{G}} \cdot 10^{3}}{\varepsilon \cdot \mathrm{t} \cdot \mathrm{V}_{\mathrm{S}}} \mathrm{U} / l .
$$

Dabei sind $\Delta \mathrm{E}=$ Differenz von Haupt- und Leerwert $\varepsilon=$ millimolarer Extinktionskoeffizient; $t=$ Inkubationszeit in Min.;
$V_{G}=$ Gesamtvolumen nach Reagenzzugabe und $V_{S}=$ Volumenanteil des Serums vom Inkubationsansatz in $\mathrm{m} l$.

Für die oben beschriebene Arbeitweise ergibt sich demnach:

Bei Messung obne $\mathrm{Mg}^{2+}-\mathrm{Zusatz}$ : Enzymkonzentration $=\Delta \mathrm{E} \cdot 10,41 \mathrm{U} / l$;

bei optimaler $\mathrm{Mg}^{2+}$-Aktivierung: Enzymkonzentration $=\Delta \mathrm{E} \cdot 36,5 \mathrm{U} / l$.

Die Enzymaktivität sollte prinzipiell bei $25^{\circ}$ bestimmt werden (33). Geschieht dies bei niedrigerer (Zimmer-) Temperatur, so lassen sich mit Hilfe von $\mathrm{Q}_{10}=2,3$ (ohne Zusatz von $\mathbf{M g}^{2+}$-Ionen) und $\mathrm{Q}_{10}=2,2$ (bei optimaler $\mathrm{Mg}^{2+}$-Aktivierung) nach der Formel (34)

$$
\log F=\frac{25-t}{10} \cdot \log Q_{10}
$$

die Umrechnungsfaktoren $\mathrm{F}_{\mathbf{1}}$ (ohne Zusatz von $\mathrm{Mg}^{2+}$-Ionen) und $\mathrm{F}_{2}$ (bei optimaler $\mathrm{Mg}^{2+}$-Aktivierung) errechnen, mit denen man die bei $t^{\circ}$ ermittelten Aktivitäten auf $25^{\circ}$ umrechnen kann (s. Tab. 3).

Tab. 3

Faktoren $\left(F_{1}\right.$ und $\left.F_{2}\right)$ zur Umrechnung von bei Zimmertemperaturen $\left(t^{\circ}\right)$ gemẹssenen Aktivitäten auf $25^{\circ}$

\begin{tabular}{ccc}
\hline $\mathbf{t}^{\circ}$ & $\mathbf{F}_{\mathbf{1}}$ & $\mathbf{F}_{\mathbf{2}}$ \\
\hline 20 & 1,52 & 1,48 \\
21 & 1,40 & 1,37 \\
22 & 1,28 & 1,27 \\
23 & 1,18 & 1,17 \\
24 & 1,09 & 1,08 \\
25 & 1,00 & 1,00 \\
\hline
\end{tabular}

\section{Ergebnisse}

\section{Normalwerte}

Es wurde Serum und Plasma von Blutspendern in der beschriebenen Weise untersucht (Tab. 4). Wie aus der Abbildung $6 \mathrm{zu}$ ersehen ist, weisen sowohl die Serumals auch die Plasmawerte ohne und mit Aktivierung eine log-normale Verteilung auf. Die höhere Aktivitä̈t im Serum im Vergleich zum Plasma (ohne $\mathrm{Mg}^{2+}$-Zusatz)

Tab. 4

\begin{tabular}{|c|c|c|c|c|c|}
\hline & \multicolumn{2}{|c|}{$\begin{array}{l}\text { Anzahl der } \\
\text { Blutspender }\end{array}$} & \multirow[b]{2}{*}{$\log \overline{\mathbf{x}}$} & \multirow[b]{2}{*}{$\log s$} & \multirow{2}{*}{$\begin{array}{c}\text { Unterer und } \\
\text { oberer Grenzwert } \\
\text { (95\% Vertrauens- } \\
\text { bereich) U/l }\end{array}$} \\
\hline & Männer & Frauen & & & \\
\hline $\begin{array}{l}\text { Serum } \\
\text { pH 10,0 }\end{array}$ & 48 & 47 & $0,9412-1$ & 0,1657 & $0,417-1,87$ \\
\hline $\begin{array}{l}\text { Plasma } \\
\text { pH } 10,0\end{array}$ & 24 & 23 & $0,7553-1$ & 0,2116 & $0,22-1,51$ \\
\hline $\begin{array}{l}\text { Plasma } \\
\text { pH 9,25 } \\
\text { Mg2+-Akt. }\end{array}$ & 24 & 25 & 0,2945 & 0,151 & $-3,95$ \\
\hline
\end{tabular}

Normalwerte der LAP in Serum und Plasma von Blutspendern

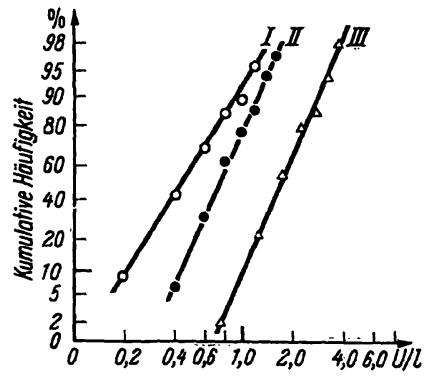

Abb. 6

Verteilung der Normalwerte der LAP-Aktivität im I Plasma $\mathrm{pH} 10,0$

III Plasma $\mathrm{pH} 9,25$, Aktivierung mit $4 \mathrm{mM} \mathrm{Mg} \mathrm{Mg}^{2+}$ Darstellung im Wahrscheinlichkeitsnetż mit logarithmischer Abszissen-
teilung 
ist wahrscheinlich auf die Freisetzung von LAP aus den Thrombocyten bei der Gerinnung zurückzuführen. Die LAP-Aktivitäten von Thrombocyten und Serum verhalten sich nach früheren Untersuchungen (35) etwa wie $500: 1$. Die Unterschiede der Enzymaktivitäten bei Männern und Frauen sowie bei verschiedenen Altersgruppen waren nicht signifikant.

\section{Experimentelles}

Vergleich der Hydrolyse von L-Leucinamid und DL-Leucinbydrazid

Abbildung 7 stellt die Hydrolyse von L-Leucinamid und DL-Leucinhydrazid durch Nörmalserum und Modellserum als Funktion des $\mathrm{pH}$ dar. pH-Optima und Umsatzraten stimmen für beide Substrate nahezu überein (s. Tab. 5). In beiden Fällen wird das Enzym durch $4 \mathrm{mM} \mathrm{Mg}^{2+}$-Ionen um das 4 fache aktiviert. Der bei pH 7,0-7,5 auftretende niedrigere Gipfel ist auf eine Beimengung von Arylamidase zurückzuführen.

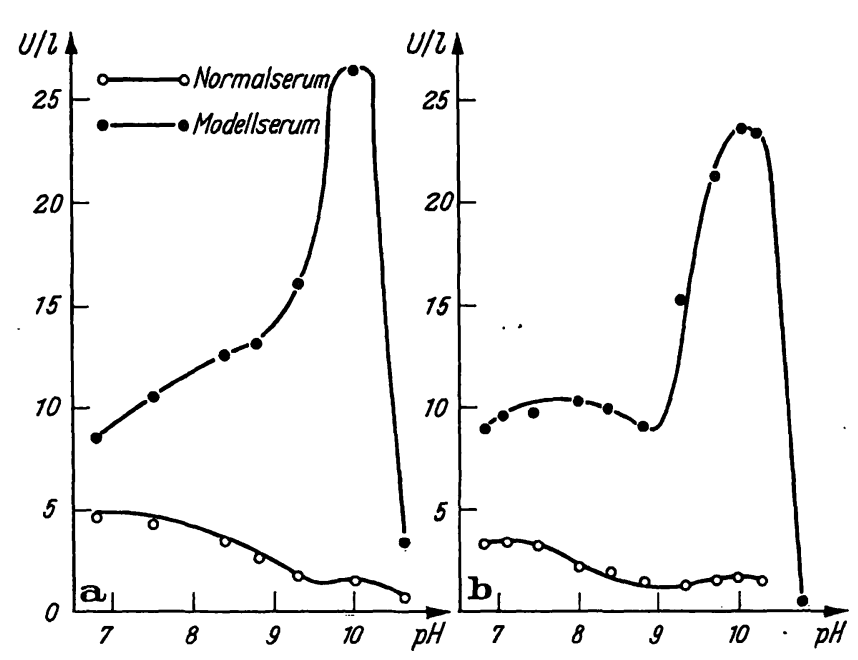

Abb. 7

Vergleichende Hydrolyse von L-Leucinamid und DL-Leucinhydrazid in Abhängigkeit vom pH ○— Normalserum, -— Modellserum (9,95 Tle. Serum + 0,05 Tle. LAP-Eluat) $\begin{array}{ll}\text { a) und c) Substrat: L-Leucinamid (16 mss); } & \text { b) und d) Substrat: DL-Leucinhydrazid (16 ms, bezogen auf L-Komponente); a) und b) ohne, } \\ \text { c) und d) mit Aktivierung durch } \mathrm{Mg}^{2+}-\mathrm{I} \text { ) } & \text { anen (4 mM). }\end{array}$ Bei Verwendung von L-Leucinamid als Substrat erfolgte die Auswertung mittels quantitativer Papierchromatographie (16-18)
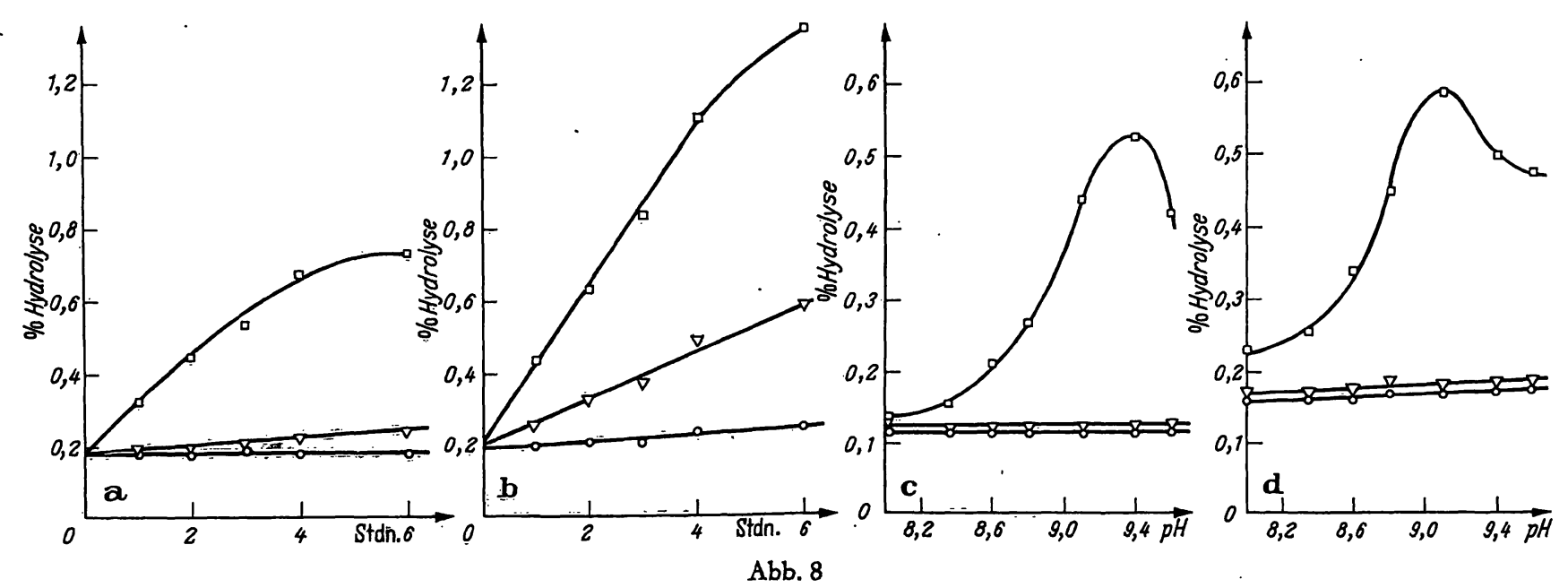

Hydrolyse von $32 \mathrm{mM}$ DL-Leucinhydrazid in wäßriger Lösung in Gegenwart von Aktivatoren der LAP

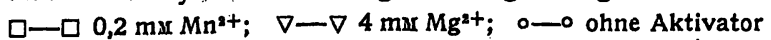

a) und b) als Funktion der Zeit; c) und d) als Funktion des $\mathrm{pH}$; Inkubation: a) und c) bei $25^{\circ}$, b) und d) bei $37^{\circ}$ 
fersubstanzen wurden selbst hergestellt (s. o.). Ein zuerst verwendetes kommerzielles Produkt enthielt etwa 0,1\% Mn. Geeignet sind die Produkte der Fa. Feinchemie K.-H. Kallies K. G., Sebnitz (Sachsen).

\section{Nicbtenz ymatische Hydrolyse von Leucinbydrazid}

Bei der Überprüfung der Aktivierbarkeit der LAP durch $\mathrm{Mg}^{2+}$ bzw. $\mathrm{Mn}^{2+-I o n e n ~ z e i g t e ~ s i c h, ~ d a ß ~} \mathrm{Mn}^{2+}$ für die
Aktivierung bei dieser Methode nicht geeignet ist. Die nichtenzymatische Hydrolyse von Leucinhydrazid wird durch $\mathrm{pH}$-Änderungen im praktisch wichtigen Bereich kaum beeinflußt. Mit steigender Temperatur nimmt die Hydrolyse deutlich zu. Manganionen wirken sich unter allen Umständen nachteilig aus. Dagegen ist der Effekt von Magnesiumionen vergleichsweise gering (Abb. 8). Aus diesem Grunde wurde in weiteren Versuchen nur
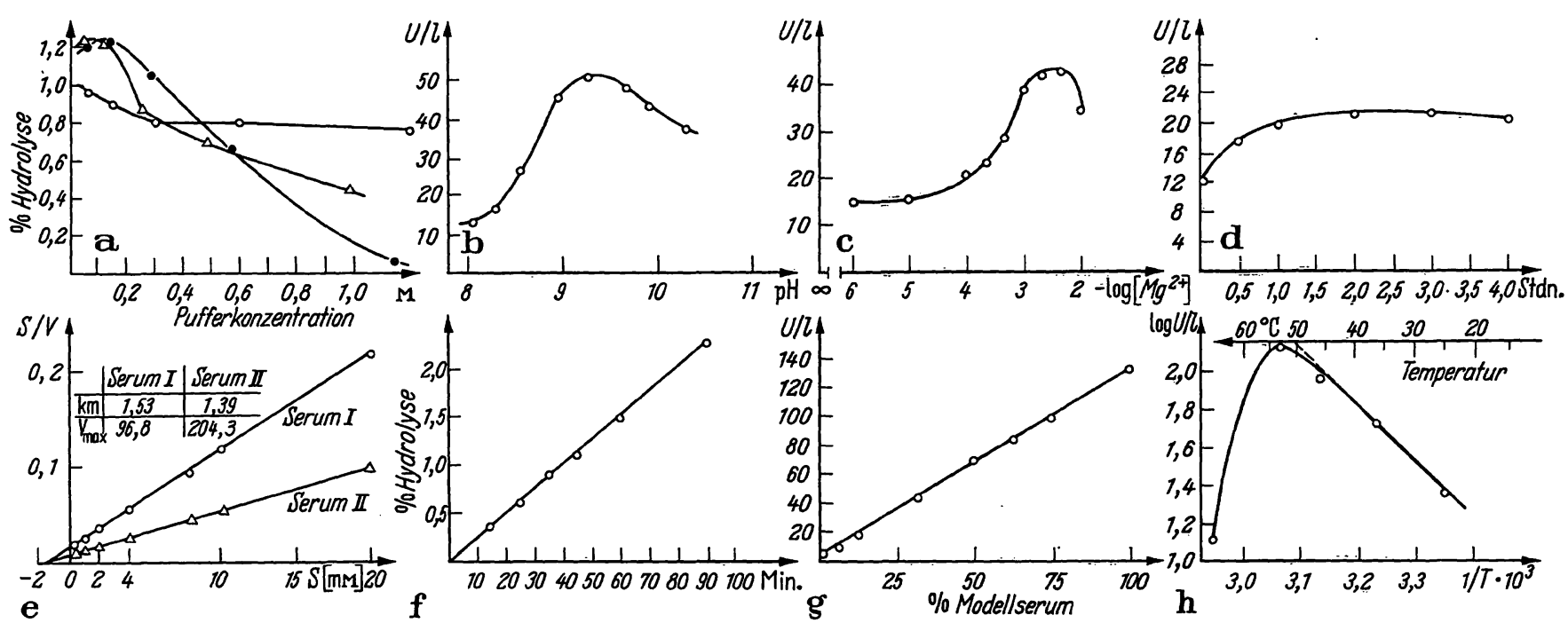

Abb. 9

LAP-Aktivität bei $\mathrm{Mg}^{2+}$-Aktivierung in Abhängigkeit

a) von der Pufferkonzentration, - - Monoäthanolamin-HCl/NaOH; 0 - Tris/ $\mathrm{HCl} ; \Delta-\Delta$ Triäthanolamin-HCl/NaOH; b) vom pH-Wert; c) von der Aktivatorkonzentration; d) von der Präinkubationsdauer; e) von der Substratkonzentration (Darstellung nach LINEWEAvER-
BURK [36]) bei zwei verschiedenen Enzymkonzentrationen (80- und 160 fache LAP-Aktivität vom Normalserum); f) von der Inkubationsdauer (Enzymaktivität in \% Hydrolyse L-Leucinhydrazid, $1 \%$ Hydrolyse $\triangleq \Delta \mathrm{E}_{455}^{1} \mathrm{~cm}$ von 0,91 ); g) von der Enzymkonzentration (Verdünnung eines 170 fach erhöhten Modellserums mit Normalserum, Abszisse: Vol. \% Modellserum); h) von der Temperatur (Darstellung nach ARRHENIUS [37]).

Inkubationsbedingungen für alle Parameter außer dem jeweils variablen: Puffer: 0,125Ms Monoäthanolamin pH 9,25; Aktivierung: 4 mær Mg2+ 2 Stdn. Präinkubation; Substrat: $32 \mathrm{mss}$ DL-Leucinhydrazid; Inkubationstemperatur: $25^{\circ}$
;
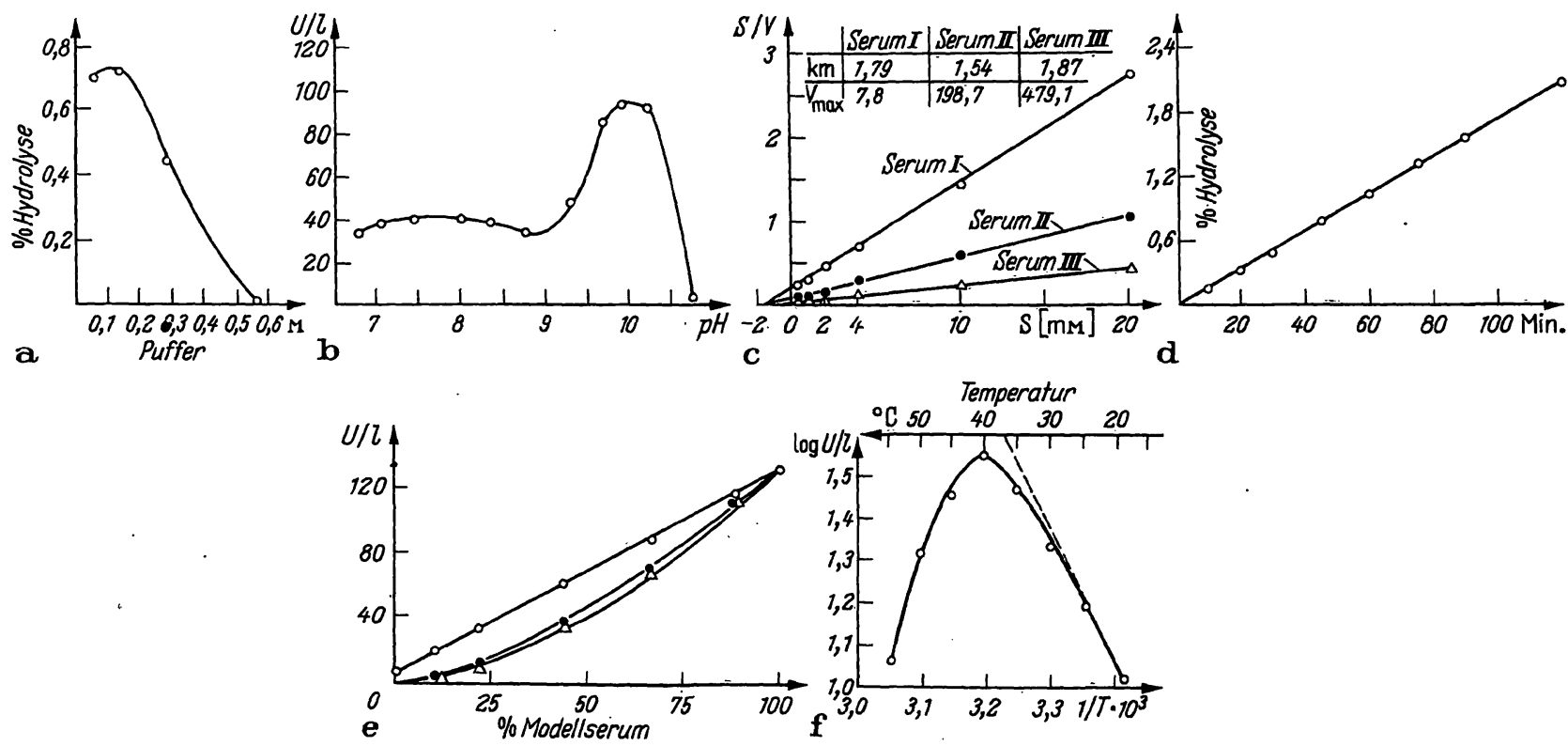

Abb. 10

LAP-Aktivität ohne Zusatz von Metallionen in Abhängigkeit

a) von der Pufferkonzentration (Monoäthanolamin-HCl/NaOH); b) vom pH-Wert; c) von der Substratkonzentration (Darstellung nach LINEWEAVER-BURK [36]) bei drei verschiedenen Enzymkonzentrationen (10-, 25- und 60 fache LAP-Aktivität vom Normalserum); d) von der Inkubationsdauer (Enzymaktivität in \% Hydrolyse L-Leucinhydrazid, $1 \%$ Hydrolyse $\Delta \Delta_{455}^{1} \mathrm{~cm}$ von 1,0 ); e) von der Enzymkonzentration (Verdünnung eines $200-$ fach erhöhten Modellserums mit Normalserum $\circ-0$; mit $0,9-$ proz. NaCl-Lösung $\bullet-\bullet$, mit aqua bidest. $\Delta-\Delta$; Abszisse: Vol.\% Modellserum); f) von der Inkubationstemperatur [Darstellung nach ARRHENIUS (37)]:

Inkubationsbedingungen für alle Parameter außer dem jeweils variablen: Puffer: 0,114m Monoäthanolamin pH 10,0; Substrat: 32 mu DLLeucinhydrazid; Inkubationstemieratur: $25^{\circ}$ 
Magnesium als Aktivator verwandt. Úber die Aufspaltung von Peptidbindungen durch Schwermetallionen, wie z. B. $\mathrm{Cer}^{3+-}, \mathrm{Cer}^{4+-}$ und $\mathrm{Th}^{4+-I o n e n}$ wurde früher von BAMANN und Mitarbeitern (36) berichtet.

\section{Enz.ymstabilität}

Die Enzymaktivität ist im Serum bei Aufbewahrung desselben im Kühlschrank mindestens bis $48 \mathrm{Stdn}$. konstant.

\section{Optimierung der Bestimnumg mit $\mathrm{Mg}^{2+}-$ Aktivierung}

Die bei der Untersuchung der einzelnen Parameter erhaltenen Ergebnisse zeigt Abbildung 9. Danach ergeben sich für eine Bestimmung der LAP-Aktivität im Serum mit $\mathrm{Mg}^{2+}$-Zusatz folgende optimale Bedingungen: Monoäthanolamin- $\mathrm{HCl} / \mathrm{NaOH}-\mathrm{Puffer}$, $0,125 \mathrm{M}$ (final), pH 9,25; $4 \mathrm{~mm} \mathrm{Mg}{ }^{2+}, 2 \mathrm{Stdn}$. Präinkubation bei $25^{\circ}$; Substratkonzentration: $16 \mathrm{mM}\left(\mathrm{K}_{\mathrm{m}}=1,5 \mathrm{mM}\right.$, Mittel aus zwei Bestimmungen); Maximale Spaltung bei $56^{\circ}$, Temperatur-Optimum zwischen $30^{\circ}$ und $40^{\circ}, Q_{10}=2,2$. Die Bestimmung der Serum-LAP mit $\mathrm{Mg}^{2+}$-Aktivierung erschien für Routinezwecke weniger geeignet, da die notwendige Präinkubation einen größeren Zeitaufwand bedingt. Deshalb wurde auch eine Methode zur Bestimmung der Serum-LAP ohne Aktivierung ausgearbeitet.

\section{Optimierung der Bestimmung obne $\mathrm{Mg}^{2+}-Z u s a t z$}

In Abbildung 10 sind die wichtigsten Parameter zusammengestellt. Danach ergeben sich folgende optimale Bedingungen: Monoäthanolamin- $\mathrm{HCl} / \mathrm{NaOH}-\mathrm{Puffer}$ 0,114 $\mathrm{M}, \mathrm{pH} 10,0$; Substratkonzentration: $16 \mathrm{mM}\left(\mathrm{K}_{\mathrm{m}}=\right.$ 1,7 mM, Mittel von 3 Bestimmungen); Maximale Spaltung bei $40^{\circ}$, Temperatur-Optimum bei $25^{\circ}, \mathrm{Q}_{10}=2,3$. Das auf der Abbildung $10 \mathrm{~b}$ im pH-Bereich 7,5 auftretende flache Optimum ist wiederum der Arylamidase zuzuschreiben. Abbildung $10 \mathrm{~d}$ zeigt im gemessenen Bereich (bis $1,8 \%$ Hydrolyse) einen linearen Verlauf der Zeit-Umsatz-Kurve.

Zur Ermittlung der Beziehungen zwischen Meßgröße und Enzymkonzentration wurde ein mit Leber-LAP an- gereichertes Serum mit Normalserum verdünnt. Es ergab sich eine lineare Korrelation. Bei Verdünnung des LAP-reichen Serums mit bidest. Wasser oder 0,9 proz. $\mathrm{NaCl}-L o ̈ s u n g$ weichen die Kurven von der Geraden ab (Abb. 10e). Daraus folgt, daß bei dieser Bestimmungsmethode das Serum nicht mit Wasser oder physiolog. $\mathrm{NaCl}-\mathrm{L}$ ösung verdünnt werden darf, da bei $\mathrm{pH}$ 10,0 mit zunehmender Verdünnung auch die Denaturierung stärker wird.

\section{Zuverlässigkeit der Methode $(39,40)$}

Spezifität: Die spezifische Bestimmung der Serum-LAP setzt eine deutliche Abgrenzung von der gleichfalls im Serum vorhandenen Arylamidase voraus. Diese vermag auch Substrate wie Leucinamid und Leucinhydrazid zu hydrolysieren (9). Durch ein abweichendes pH-Optimum $(7,0)$ und die fehlende Aktivierbarkeit bzw. eine Hemmung durch $\mathrm{Mn}^{2+}$ - und $\mathrm{Mg}^{2+-}$ Ionen läßt sie sich aber weitgehend von der LAP abgrenzen (s. Abb. 7).

Ricbtigkeit: Bei Mischversuchen mit Modell-Seren von hoher Aktivität und Normalserum ergab sich ein Korrelationskoeffizient von 0,998 bzw. 0,999 (Abb. $9 \mathrm{~g}$ und 10e).

Wiederbolbarkeit: Bei jeweils 10 Bestimmungen wurden an der oberen Normgrenze (1,9 U/l bzw. 4,0 U/l) folgende Variationskoeffizienten gefunden: Bestimmung im Serum obne Zusatz von $\mathrm{Mg}^{2+}-$ Ionen: $\mathrm{V}_{\mathrm{k}}=1,9 \%$; Bestimmung im Plasma mit optimaler $\mathrm{Mg}^{2+}$-Aktivierung $\mathrm{V}_{\mathbf{k}}=2,1 \%$.

Empfindlicbkeit: $\mathrm{Da}$ der Variationskoeffizient an der oberen Grenze der Norm niedrig ist, ist die Empfindlichkeit der Methode entsprechend hoch.

Für die Úberlassung der elektrophoretisch isolierten LAP sowie der Abbildung 4 sind wir Herrn Dipl.-Chem. N. REHFELD zu besonderem Dank verpflichtet.

Wir danken dem Bezirksinstitut für Blutspende- und Transfusionswesen (Direktor: Dr. med. W. SchUBERT) für die Bereitstellung der Blutproben und den Herren Dr. H. Jeschkert (Institut für organische Chemie) und Dr. R. MASThoff (Institut für anorganische Chemie) für die Anfertigung von Elementar- und spektrographischen Analysen.

Für technische Assistenz danken wir Frau G. Henning.

\section{Literatur}

1. HAschen, R. J., Proteolytische Enzyme bei Leberkrankheiten, Symposium über enzymologische Diagnostik der Lebetkrankheiten, 27.-28.11.1964, Warschau. - 2. Fletsher, G. A., M. Pankow und C. Warmka, Clin. chim. Acta (Amsterdam) 9, 259 (1964). - 3. HASCHEN, R. J., W. FARR, H. GIESECKE und D. Reichelt, in Vorbereitung. - 4. SMITH, E. L. und D. H. Spackmann, J. biol. Chemistry 212, 271 (1955). - 5. Patterson, E. K., A. Keppei und S. H. HsiaO, J. Histochem. Cytochem. 9, 609 (1961). - 6. Patterson, E. K., S. H. Hsiao und A. Keppex, J. biol. Chemistry 238, 3611 (1963). - 7. Pfleiderer, G., P. G. Celliers, M. Stanulovic, E. D. Wachismuth, H. DetermanN und G. Braunttzer, Biochem. Z. 340, 552 (1964). - 8. MANNSFeLdT, H.-G., H.-J. HütTeR und H. HaNson, Zur Darstellung einer Aminopeptidase aus Mikrosomen und Mitochondrien von Schweinenieren, 3. Jahrestagung der. Biochemi- schen Gesellschaft der DDR, 18.-20. 9. 1966, Suhl/Thür. - 9. HASChEN, R. J., N. REhfeld und H. Giesecke, Naturwissenschaften 53, 434 (1966). - 10. GöGGeL, K. H., W. CREutzFeLdT und J. Murucas, Dtsch. med. Wschr. 85, 1756, 1808 (1960). 11. Tuppy, H., U. Wiesbauer und E. Wintersberger, HoppeSeyler's Z. physiol. Chem. 329, 278 (1962). - 12. GoldBerG, J. A., E. P. Pineda und A. M. Rutenburg, Amer. J. clin. Pathol. 32, 571 (1959). - 13. Nagel, W., F. Wildig, W. Peschke und F. H. Schmidt, Hoppe-Seylers' Z. physiol. Chem. 340, 1 (1965). - 14. GrassmanN, E. und W. Heyde, Hoppe-Seyler's Z. physiol. Chem. 183, 32 (1929). - 15. LinderstrøMr-LANG, K., Hoppe-Seylers' Z. physiol. Chem. 173, 32 (1928). - 16. Haschen, R. J., Clin. chim. Acta (Amsterdam) 1, 242 (1956). 17. Haschen, R. J., Clin. chim. Acta (Amsterdam) 6, 322 (1961). - 18. Haschen, R. J., Biochem. Z. 334, 569 (1961). - 19. 
Hanson, H. und R. J. Haschen, Hoppe-Seyler's Z. physiol. Chem. 310, 213 (1958). - 20. NAGed, W., F. WiLlig und F. H. Schmidr, Klin. Wschr. 42, 447 (1964). - 21. Fleisher, G. A., M. Pankow und C. WARnKa, Clin. chim. Acta (Amsterdam) 9, 254 (1964). - 22. Knöpfer, M., Mh. Chem. 30, 31 (1909). - 23. Pesez, M. und A. Petri, Bull. soc. chim. France 1947, 122. - 24. Watt, G. W. und J. D. Chrisp, Analytic. Chem. 24, 2006 (1952). - 25. Thies, H. und G. Kallinich, Biochem. Z. 324, 485 (1953). - 26. Yale, H. L., K. Losee, J. Martius, H. Holsing, F. M. Perry und J. Bernstein, J. Amer. chem. Soc. 75, 1933 (1953). - 27. Losse, G., H.-J. Hebel und CH. KästNer, J. prakt. Chem. 8, 339 (1959). - 28. Kästner, CH., Diplomarbeit, Mat.-Nat. Fak., Halle/Saale, 1959. - 29. Chambers, R. A. und F. H. Carpenter, J. Amer. chem. Soc. 77, 1522 (1955). - 30. Wergin, A., Naturwissenschaften 52, 34 (1965). - 31. Kallos, J.,
D. KaHN und D. Rizok, Canad. J. Biochem. 42, 235 (1965). 32. Kallos, J., E. L. Arthur, D. RizoK und D. Kahn, Canad. J. Biochem. 43, 135 (1965). - 33. Report of the Comission on Enzymes of the International Union of Biochemistry, Pergamon Press, Oxford 1961. - 34. Aebr, H., in: Rauen, H. M. (Hrsg.): „Biochemisches Taschenbuch“, 2. Teil, S. 167, Springer-Verlag, Berlin-Göttingen-Heidelberg-New York 1964. - 35. BeESE, J., W. FARR, E. GRÜNER und R. J. HASChEN, Klin. Wschr. 44, 1049 (1966). - 36. Bamann, E., J. G. HaAs und H. Trappmann, Naturwissenschaften 46, 73 (1959). - 37. LINEWEAvER, H. und D. BuRK, J. Amer. chem. Soc. 56, 658 (1934). - 38. NeTrer, H., Theoretische Biochemie, Springer-Verlag, Berlin-Göttingen-Heidelberg 1959. - 39. Hughes, H. K., Analytic. Chem. 24, 1349 (1952). 40. Richterich, R., Klinische Chemie, S. 52, S. Karger, BaselNew York 1965.

\title{
Zur Immunchemischen Bestimmung von Enzymproteinen der Alkoholdehydrogenase mit Hilfe der Nephelometrie ${ }^{1}$ )
}

\author{
Yon T. O. KLEINE \\ Aus dem Physiologisch-Chemischen Instituț der Universität Hamburg (Direktor: Prof. Dr. J. Kübnau)
}

(Eingegangen am 4. März 1967)

\begin{abstract}
Mit Hilfe von präzipitierenden, auf Alkoholdehydrogenase aus Hefe und Pferdeleber spezifischen Antikörpern wird ein Mikrotest beschrieben, worin die Enzymprotein-Antikörper-Trübung nephelometrisch serienmäßig bestimmt wird. Im Hefehomogenat sowie im Überschuß an Fremdprotein (menschliches Blutserum) kann mit dieser Methode in einem Gesamtvolumen von 0,40 ml 0,3-0,6 $\mu \mathrm{g}$ und mehr Enzymprotein mit einem mittleten Fehler von $\pm 4 \%$ gemessen werden.
\end{abstract}

Precipitating antibodies to alcoholdehydrogenase (Alcohol: NAD oxidoreductase E. C. 1.1.1.1) from yeast and horse liver were used in a microtest for serial measurement of the turbidity caused by the enzyme-protein-antibody-reaction in vitro. With this method and a mean error of $\pm 4 \%, 0,3$ to $0,6 \mu \mathrm{g}$ of enzyme protein and more per $0,40 \mathrm{ml}$ can be measured in yeast homogenate as well as in the presence of excess foreign protein (human blood serum).

Enzyme werden im allgemeinen mit Hilfe ihrer Reaktionsgeschwindigkeit (Aktivität) unter definierten Standardbedingungen gemessen. Hierbei wird die Enzymquantität nur insofern berücksichtigt, als diejenige Enzymmenge bestimmt wird, die $1 \mu \mathrm{Mol}$ Substrat pro Min. unter jenen Bedingungen umsetzt (1). Für die heutige Enzymchemie sind jene Meßbedingungen bei der Bestimmung von allosterisch oder induktiv beeinflußter Enzymaktivität ungenügend: Eine z. B. in einem Gewebehomogenat erhöhte oder verminderte Enzymaktivität kann auf eine quantitative Vermehrung oder Verminderung des Enzymproteins oder aber auf eine allosterische Steigerung oder Hemmung der Enzymaktivität zurückgeführt werden. Beide Phänomene lassen sich durch die Messung der Reaktionsgeschwindigkeit unter Standardbedingungen nicht voneinander unterscheiden.

Mit Hilfe des nephelometrisch gemessenen AntigenAntikörperkomplexes, einer für quantitative Messungen von Proteinen des Blutes (2-9) bereits bewährten

1) Mit Unterstützung der Deutschen Forschungsgemeinschaft.
Methode haben wir versucht, einen Test zur quantitativen Messung des Enzymproteins aufzubauen, mit dem Enzymprotein im Homogenat oder in Gegenwart hoher Fremdproteinkonzentrationen gemessen werden kann. Durch die simultane Messung von Menge und Aktivität eines Enzyms ist es u. E. möglich, genauere Aussagen über dieses Enzym zu machen als es die bisherigen Methoden erlaubten.

\section{Methodik}

Erzeugung von Antikörpern auf Alkoholdehydrogenas $\mathrm{e}^{1}$ ) aus Hefe und Pferdeleber

$2 \mathrm{~kg}$ schweren, männlichen Kaninchen (Neuseeländer Albinos) werden mit eintägigem Intervall aufeinanderfolgend $0,5,1,0,1,5$, 2,0 und 4,0 mg Enzymprotein Hefe-Alkoholdehydrogenase (HADH) bzw. Alkoholdehydrogenase aus Pferdeleber (LADH) ${ }^{2,3}$ )

1) Der Trivialname Alkoholdehydrogenase wird hier gebraucht für das Enzym Alkohol: NAD oxidoteductase EC 1.1.1.1.

2) Bezogen von C. F. Boehringet, Mannheim.

3) Abkürzungen: $\mathrm{HADH}=$ Hefe-Alkoholdehydrogenase; $\mathrm{LADH}$ = Leber-Alkoholdehydrogenase; EDTA = Athylendiamintetraessigsäure. 